\title{
Bevacizumab/Liposomal Doxorubicin Regimen
}

National Cancer Institute

\section{Source}

National Cancer Institute. Bevacizumab/Liposomal Doxorubicin Regimen. NCI

Thesaurus. Code C160525.

A reg imen consisting of bevacizumab and liposomal doxorubicin that can be used for the treatment of ovarian, fallopian tube, and primary peritoneal cancers. 